\title{
Observation of Complex Time Structures in the Cosmic-Ray Electron and Positron Fluxes with the Alpha Magnetic Spectrometer on the International Space Station
}

M. Aguilar, ${ }^{27}$ L. Ali Cavasonza, ${ }^{1}$ G. Ambrosi, ${ }^{32}$ L. Arruda,${ }^{25}$ N. Attig, ${ }^{22}$ S. Aupetit, ${ }^{18}$ P. Azzarello, ${ }^{17}$ A. Bachlechner, ${ }^{1}$ F. Barao ${ }^{25}$ A. Barrau, ${ }^{18}$ L. Barrin, ${ }^{16}$ A. Bartoloni,,${ }^{37}$ L. Basara, ${ }^{35}$ S. Başeğmez-du Pree, ${ }^{6}$ M. Battarbee, ${ }^{45}$ R. Battiston,,${ }^{35,36, a}$ U. Becker, ${ }^{10}$ M. Behlmann, ${ }^{10}$ B. Beischer, ${ }^{1}$ J. Berdugo, ${ }^{27}$ B. Bertucci, ${ }^{32,33}$ K. F. Bindel,${ }^{23}$ V. Bindi, ${ }^{20}$ W. de Boer, ${ }^{23}$ K. Bollweg, ${ }^{21}$ V. Bonnivard, ${ }^{18}$ B. Borgia, ${ }^{37,38}$ M. J. Boschini, ${ }^{29}$ M. Bourquin, ${ }^{17}$ E. F. Bueno, ${ }^{39}$ J. Burger, ${ }^{10}$ F. Cadoux, ${ }^{17}$ X. D. Cai, ${ }^{10}$ M. Capell, ${ }^{10}$ S. Caroff, ${ }^{3}$ J. Casaus,${ }^{27}$ G. Castellini, ${ }^{15}$ F. Cervelli, ${ }^{34}$ M. J. Chae, ${ }^{40}$ Y. H. Chang, ${ }^{11}$ A. I. Chen, ${ }^{10}$ G. M. Chen, ${ }^{6}$ H. S. Chen, ${ }^{6,7}$ Y. Chen,${ }^{17}$ L. Cheng, ${ }^{41}$ H. Y. Chou, ${ }^{11}$ E. Choumilov ${ }^{10}$ V. Choutko, ${ }^{10}$ C. H. Chung, ${ }^{1}$ C. Clark, ${ }^{21}$ R. Clavero, ${ }^{24}$ G. Coignet, ${ }^{3}$ C. Consolandi, ${ }^{20}$ A. Contin, ${ }^{8,9}$ C. Corti, ${ }^{20}$ W. Creus, ${ }^{44}$ M. Crispoltoni, ${ }^{32,33}$ Z. Cui, ${ }^{41}$ K. Dadzie, ${ }^{10}$ Y. M. Dai ${ }^{5}$ A. Datta,${ }^{20}$ C. Delgado, ${ }^{27}$ S. Della Torre, ${ }^{29}$ M. B. Demirköz, ${ }^{2}$ L. Derome, ${ }^{18}$ S. Di Falco, ${ }^{34}$ F. Dimiccoli,,${ }^{35,36}$ C. Díaz, ${ }^{27}$ P. von Doetinchem, ${ }^{20}$ F. Dong, ${ }^{31}$ F. Donnini, ${ }^{32,33}$ M. Duranti, ${ }^{32}$ D. D’Urso, ${ }^{32, b}$ A. Egorov, ${ }^{10}$ A. Eline, ${ }^{10}$ T. Eronen, ${ }^{45}$ J. Feng, ${ }^{10}$ E. Fiandrini, ${ }^{32,33}$ P. Fisher, ${ }^{10}$ V. Formato, ${ }^{32}$ Y. Galaktionov, ${ }^{10}$ G. Gallucci, ${ }^{34}$ R. J. García-López ${ }^{24}$ C. Gargiulo, ${ }^{16}$ H. Gast, ${ }^{1}$ I. Gebauer, ${ }^{23}$ M. Gervasi,${ }^{29,30}$ A. Ghelfi, ${ }^{18}$ F. Giovacchini, ${ }^{27}$ D. M. Gómez-Coral, ${ }^{28}$ J. Gong, ${ }^{31}$ C. Goy, ${ }^{3}$ V. Grabski, ${ }^{28}$ D. Grandi, ${ }^{29}$ M. Graziani, ${ }^{23}$ K. H. Guo, ${ }^{19}$ S. Haino, ${ }^{44}$ K. C. Han, ${ }^{26}$ Z. H. He, ${ }^{19}$ M. Heil, ${ }^{10}$ T. H. Hsieh, ${ }^{10}$ H. Huang, ${ }^{44, \mathrm{c}}$ Z. C. Huang, ${ }^{19}$ C. Huh, ${ }^{14}$ M. Incagli, ${ }^{34}$ M. Ionica, ${ }^{32}$ W. Y. Jang, ${ }^{14}$ Yi Jia, ${ }^{10}$ H. Jinchi, ${ }^{26}$ S. C. Kang, ${ }^{14}$ K. Kanishev, ${ }^{35,16}$ B. Khiali, ${ }^{11}$ G. N. Kim, ${ }^{14}$ K. S. Kim, ${ }^{14}$ Th. Kirn, ${ }^{1}$ C. Konak, ${ }^{2}$ O. Kounina, ${ }^{10}$ A. Kounine, ${ }^{10}$ V. Koutsenko, ${ }^{10}$ A. Kulemzin, ${ }^{10}$ G. La Vacca, ${ }^{29,30}$ E. Laudi, ${ }^{16}$ G. Laurenti, ${ }^{8}$ I. Lazzizzera, ${ }^{35,36}$ A. Lebedev, ${ }^{10}$ H. T. Lee,${ }^{43}$ S. C. Lee, ${ }^{44}$ C. Leluc, ${ }^{17}$ H. S. Li, ${ }^{42}$ J. Q. Li ${ }^{31}$ Q. Li, ${ }^{31}$ T. X. Li, ${ }^{19}$ Z. H. Li, ${ }^{6}$ Z. Y. Li ${ }^{44, d}$ S. Lim, ${ }^{14}$ C. H. Lin, ${ }^{44}$ P. Lipari, ${ }^{37}$ T. Lippert, ${ }^{22}$ D. Liu, ${ }^{11}$ Hu Liu, ${ }^{10}$ V. D. Lordello, ${ }^{39}$ S. Q. Lu, ${ }^{44,}$ Y. S. Lu, ${ }^{6}$ K. Luebelsmeyer, ${ }^{1}$ F. Luo, ${ }^{41}$ J. Z. Luo, ${ }^{31}$ S. S. Lyu,${ }^{19}$ F. Machate, ${ }^{1}$ C. Mañá, ${ }^{27}$ J. Marín, ${ }^{27}$ T. Martin, ${ }^{21}$ G. Martínez, ${ }^{27}$ N. Masi, ${ }^{8}$ D. Maurin,,${ }^{18}$ A. Menchaca-Rocha,${ }^{28}$ Q. Meng, ${ }^{31}$ V. M. Mikuni, ${ }^{39}$ D. C. Mo,${ }^{19}$ P. Mott,${ }^{21}$ T. Nelson, ${ }^{20}$ J. Q. Ni, ${ }^{19}$ N. Nikonov, ${ }^{1}$ F. Nozzoli, ${ }^{32,35, e}$ A. Oliva, ${ }^{27}$ M. Orcinha, ${ }^{25}$ M. Palermo, ${ }^{20}$ F. Palmonari, ${ }^{8,9}$ C. Palomares,${ }^{27}$ M. Paniccia, ${ }^{17}$ M. Pauluzzi,${ }^{32,33}$ S. Pensotti,${ }^{29,30}$ C. Perrina, ${ }^{17}$ H. D. Phan,${ }^{10}$ N. Picot-Clemente, ${ }^{13}$ F. Pilo, ${ }^{34}$ C. Pizzolotto, ${ }^{32, f}$ V. Plyaskin, ${ }^{10}$ M. Pohl ${ }^{17}$ V. Poireau, ${ }^{3}$ L. Quadrani, ${ }^{8,9}$ X. M. Qi ${ }^{19}$ X. Qin, ${ }^{10}$ Z. Y. Qu, ${ }^{44, g}$ T. Räihä, ${ }^{1}$ P. G. Rancoita, ${ }^{29}$ D. Rapin, ${ }^{17}$ J. S. Ricol, ${ }^{18}$ S. Rosier-Lees, ${ }^{3}$ A. Rozhkov, ${ }^{10}$ D. Rozza, ${ }^{29,30}$ R. Sagdeev, ${ }^{12}$ S. Schael, ${ }^{1}$ S. M. Schmidt, ${ }^{22}$ A. Schulz von Dratzig, ${ }^{1}$ G. Schwering, ${ }^{1}$ E. S. Seo, ${ }^{13}$ B. S. Shan, ${ }^{4}$

J. Y. Shi, ${ }^{31}$ T. Siedenburg, ${ }^{1}$ D. Son, ${ }^{14}$ J. W. Song, ${ }^{41}$ M. Tacconi,${ }^{29,30}$ X. W. Tang, ${ }^{6}$ Z. C. Tang, ${ }^{6}$ D. Tescaro, ${ }^{24}$

Samuel C. C. Ting, ${ }^{10,16}$ S. M. Ting, ${ }^{10}$ N. Tomassetti, ${ }^{32,33}$ J. Torsti, ${ }^{45}$ C. Türkoğlu, ${ }^{2}$ T. Urban, ${ }^{21}$ V. Vagelli, ${ }^{32,33}$ E. Valente,,${ }^{37,38}$ E. Valtonen, ${ }^{45}$ M. Vázquez Acosta, ${ }^{24}$ M. Vecchi,${ }^{39}$ M. Velasco, ${ }^{27}$ J. P. Vialle, ${ }^{3}$ L. Q. Wang, ${ }^{41}$ N. H. Wang, ${ }^{41}$ Q. L. Wang, ${ }^{5}$ X. Wang, ${ }^{10}$ X. Q. Wang, ${ }^{6,7}$ Z. X. Wang, ${ }^{19}$ C. C. Wei, ${ }^{44, h}$ Z. L. Weng, ${ }^{10}$ K. Whitman,${ }^{20}$ H. Wu, ${ }^{31}$ X. Wu, ${ }^{17}$ R. Q. Xiong ${ }^{31}$ W. Xu, ${ }_{10}$ Q. Yan, ${ }^{10}$ J. Yang, ${ }^{40}$ M. Yang, ${ }^{6}$ Y. Yang, ${ }^{42}$ H. Yi, ${ }^{31}$ Y. J. Yu, ${ }^{5}$ Z. Q. Yu, ${ }^{6}$ M. Zannoni, ${ }^{29,30}$ S. Zeissler, ${ }^{23}$ C. Zhang, ${ }^{6}$ F. Zhang, ${ }^{6}$ J. Zhang, ${ }^{10}$ J. H. Zhang, ${ }^{31}$ S. W. Zhang, ${ }^{6,7}$ Z. Zhang, ${ }^{10}$ Z. M. Zheng, ${ }^{4}$ H. L. Zhuang, ${ }^{6}$ V. Zhukov, ${ }^{1}$

A. Zichichi, ${ }^{8,9}$ N. Zimmermann, ${ }^{1}$ and P. Zuccon ${ }^{10}$

(AMS Collaboration)

\author{
${ }^{1}$ I. Physics Institute and JARA-FAME, RWTH Aachen University, D-52056 Aachen, Germany \\ ${ }^{2}$ Department of Physics, Middle East Technical University (METU), 06800 Ankara, Turkey \\ ${ }^{3}$ Laboratoire d'Annecy-le-Vieux de Physique des Particules (LAPP), CNRS/IN2P3 and Université Savoie Mont Blanc, \\ F-74941 Annecy-le-Vieux, France \\ ${ }^{4}$ Beihang University (BUAA), Beijing, 100191, China \\ ${ }^{5}$ Institute of Electrical Engineering (IEE), Chinese Academy of Sciences, Beijing, 100190, China \\ ${ }^{6}$ Institute of High Energy Physics (IHEP), Chinese Academy of Sciences, Beijing, 100049, China \\ ${ }^{7}$ University of Chinese Academy of Sciences (UCAS), Beijing, 100049, China \\ ${ }^{8}$ INFN Sezione di Bologna, I-40126 Bologna, Italy \\ ${ }^{9}$ Università di Bologna, I-40126 Bologna, Italy \\ ${ }^{10}$ Massachusetts Institute of Technology (MIT), Cambridge, Massachusetts 02139, USA \\ ${ }^{11}$ National Central University (NCU), Chung-Li, Tao Yuan, 32054, Taiwan \\ ${ }^{12}$ East-West Center for Space Science, University of Maryland, College Park, Maryland 20742, USA \\ ${ }^{13}$ IPST, University of Maryland, College Park, Maryland 20742, USA \\ ${ }^{14}$ CHEP, Kyungpook National University, 41566 Daegu, Korea \\ ${ }^{15}$ CNR-IROE, I-50125 Firenze, Italy
}




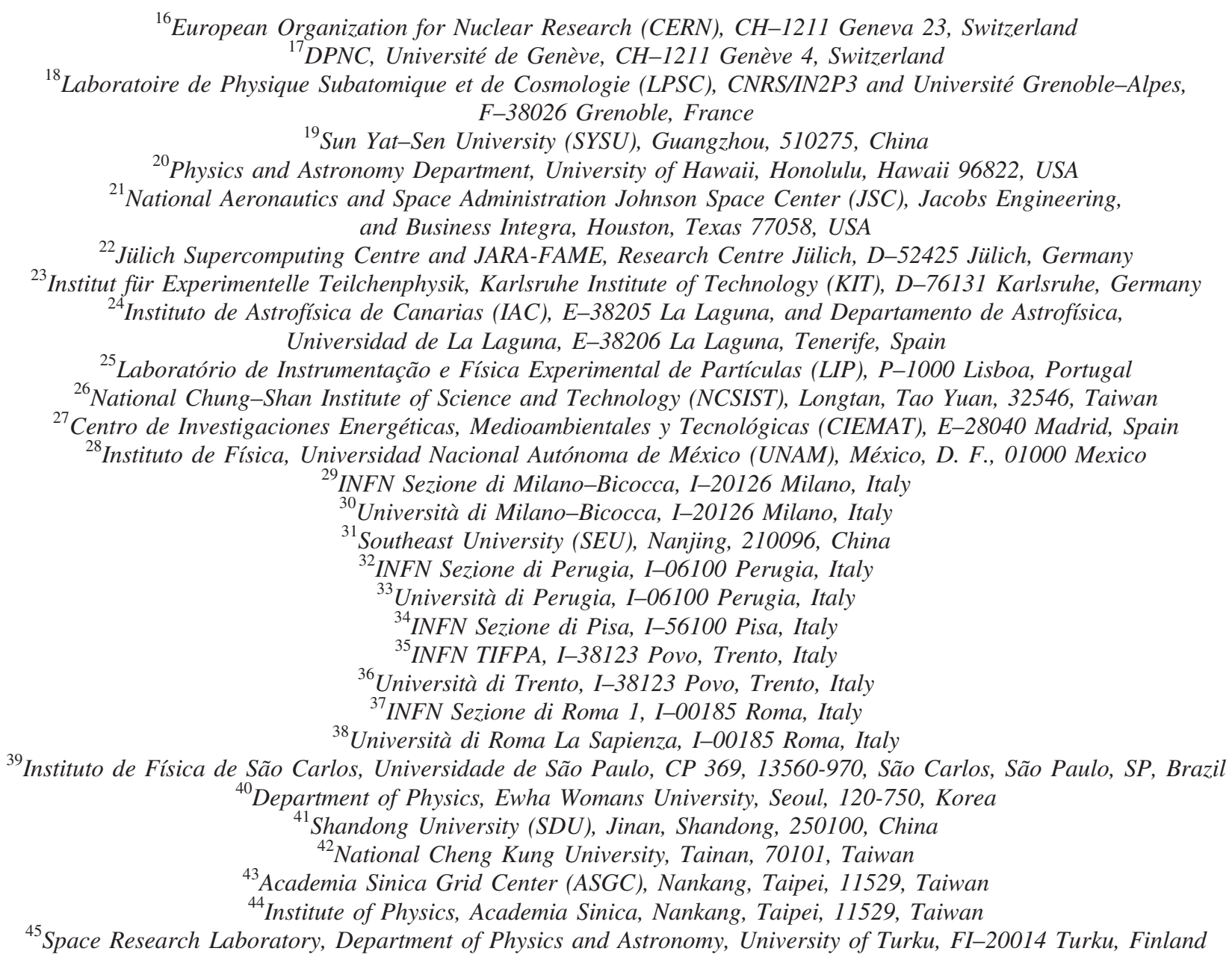

(Received 27 November 2017; revised manuscript received 11 May 2018; published 31 July 2018)

We present high-statistics, precision measurements of the detailed time and energy dependence of the primary cosmic-ray electron flux and positron flux over 79 Bartels rotations from May 2011 to May 2017 in the energy range from 1 to $50 \mathrm{GeV}$. For the first time, the charge-sign dependent modulation during solar maximum has been investigated in detail by leptons alone. Based on $23.5 \times 10^{6}$ events, we report the observation of short-term structures on the timescale of months coincident in both the electron flux and the positron flux. These structures are not visible in the $e^{+} / e^{-}$flux ratio. The precision measurements across the solar polarity reversal show that the ratio exhibits a smooth transition over $830 \pm 30$ days from one value to another. The midpoint of the transition shows an energy dependent delay relative to the reversal and changes by $260 \pm 30$ days from 1 to $6 \mathrm{GeV}$.

DOI: 10.1103/PhysRevLett.121.051102

In this Letter, we present precision measurements of the primary cosmic-ray electron flux, positron flux, and $e^{+} / e^{-}$ flux ratio $R_{e}$ in the energy range from 1 to $50 \mathrm{GeV}$ as a function of Bartels rotation (27 days), from May 2011 to

Published by the American Physical Society under the terms of the Creative Commons Attribution 4.0 International license. Further distribution of this work must maintain attribution to the author(s) and the published article's title, journal citation, and DOI.
May 2017, based on $23.5 \times 10^{6}$ electron and positron events collected by the Alpha Magnetic Spectrometer (AMS) aboard the International Space Station (ISS). These data allow comprehensive studies of the energy and charge-sign dependence of short-term effects on the time scale of months, related to solar activity $[1,2]$, and long-term effects on the time scale of years, related to the 22 year cycle of the solar magnetic field [3].

The fluxes of interstellar charged cosmic rays are thought to be stable on the time scale of decades [4-7]. 
Time-dependent structures in the energy spectra are only expected from the solar modulation [3] of interstellar cosmic rays when they enter the heliosphere. Solar modulation involves convective, diffusive, particle drift, and adiabatic energy loss processes. Only particle drift induces a dependence of solar modulation on the particle charge sign [8]. Since electrons and positrons differ only in charge sign, their simultaneous measurement offers a unique way to study charge-sign dependent solar modulation effects. Previous experiments have established that solar modulation is charge-sign dependent [9-12]. The important difference to earlier studies $[10,13-16]$ is the high statistics of the data presented in this Letter which allow for the first time precision measurements with a time resolution of one month for particles with identical mass but opposite charge sign. This measurement will continue for the entire solar cycle ( 2024) and provide a comparison to earlier measurements [16]. Our data were collected continuously during the polarity reversal of the solar magnetic field, which took place in the year 2013 [17], at the time of the solar maximum in solar cycle 24 . Therefore one expects large charge-sign dependent effects on $R_{e}$.

In addition to the long-term variations of cosmic-ray fluxes related to the solar cycle, short-term structures in the cosmic-ray proton flux [1,18] and helium flux [18] have been observed, which could occasionally be related to particular events in the solar activity. With the precision data on electrons and positrons measured simultaneously over an extended period of time, we are able to measure the difference in time-dependent structures in the fluxes of particles and antiparticles from solar effects for the first time.

The time structure of particle to antiparticle ratios like $R_{e}$ is of particular importance as these ratios have been widely used to search for new phenomena in primary cosmic rays such as the existence of a nearby positron source [19] or dark matter annihilation [20,21]. Model predictions can only be compared properly to data from long-duration experiments or experiments at different times when shortterm effects caused by the activity of the Sun and long-term effects that impact solar modulation are taken into account. Our precise data on the time and energy dependence of the electron flux and positron flux at $1 \mathrm{AU}$ provide new and additional accurate input and detailed constraints on modeling of the transport processes for charged cosmic rays inside the heliosphere [22-25]. A comprehensive model of the time-dependent solar modulation will have far-reaching consequences for the understanding of currently unexplained features in cosmic-ray fluxes, such as the observed rise of the positron fraction above $8 \mathrm{GeV}$ [26], as well as for other domains of astrophysics, such as the modeling of galactic cosmic-ray propagation [27], the estimate of the galactic cosmic-ray pressure, an important ingredient for models of galaxy formation [28], and the interpretation of possible anisotropies in the cosmic-ray arrival directions at Earth [29].
Detector.-The AMS-02 detector consists of a permanent magnet, nine planes of silicon tracker, a transition radiation detector (TRD), four planes of time-of-flight counters, an array of 16 anticoincidence counters, a ring imaging Čerenkov detector (RICH), and an electromagnetic calorimeter (ECAL). The AMS operates continuously on the ISS and is monitored and controlled continuously from the ground. A detailed description of the instrument is found in Ref. [30]. Monte Carlo simulated events were produced using a dedicated program developed by the collaboration based on the GEANT-4.10.1 package [31]. The program simulates electromagnetic and hadronic interactions of particles in the material of the AMS and generates detector responses. The Monte Carlo event samples have sufficient statistics such that they do not contribute to the errors.

Data analysis.-The data analysis follows the procedure used for our measurement of the time-averaged electron and positron fluxes [32] with improved low-energy effective acceptance [33]. The fluxes of cosmic-ray positrons and electrons for Bartels rotation $i$ in the energy bin $E$ of width $\Delta E$ are given by

$$
\Phi_{e^{ \pm}, i}(E)=\frac{N_{e^{ \pm}, i}(E)}{A_{\mathrm{eff}, i}(E) T_{i}(E) \epsilon_{\mathrm{trig}}(E) \Delta E},
$$

where $N_{e^{+}, i}$ and $N_{e^{-}, i}$ are the numbers of positrons and electrons, respectively, $A_{\text {eff, } i}$ is the effective acceptance, and $T_{i}$ is the exposure time in the given time bin. $\epsilon_{\text {trig }}$ is the trigger efficiency, which is found to be stable over time, and is $100 \%$ above $3 \mathrm{GeV}$ decreasing to $75 \%$ at $1 \mathrm{GeV}$. The same energy binning as for Ref. [32] is used up to $49.33 \mathrm{GeV}$.

The effective acceptance is defined as

$$
A_{\mathrm{eff}, i}(E)=A_{\mathrm{geom}} \epsilon(E)[1+\delta(E)]\left(1+\hat{\delta}_{i}\right),
$$

where $A_{\text {geom }}=550 \mathrm{~cm}^{2} \mathrm{sr}$ is the geometric acceptance, $\epsilon$ is the selection and identification efficiency, and the product $A_{\text {geom }} \epsilon$ is determined by Monte Carlo simulation. The timeindependent function $\delta(E)$ corrects for minor differences between simulation and data and is determined in the same way as in Ref. [32]. The absolute value of $\delta(E)$ was found to be $<4 \%$ over the entire energy range. The $\hat{\delta}_{i}$ account for small time-dependent effects in the detector response and vary at the level of $(0.0 \pm 0.4) \%$.

The exposure time $T_{i}(E)$ is determined as a function of energy for each Bartels rotation by counting the number of livetime-weighted seconds at each location above the geomagnetic cutoff [32], when the detector was in normal operating conditions and, in addition, the AMS was pointing within $40^{\circ}$ of the local zenith and the ISS was outside of the South Atlantic Anomaly. The function $T_{i}(E)$ reaches a value of $80 \%$ of a Bartels rotation at energies above $35 \mathrm{GeV}$ and smoothly declines towards lower 
energies, due to the geomagnetic cutoff. It is $6 \%$ of a Bartels rotation at $2 \mathrm{GeV}$. Below $1 \mathrm{GeV}$, the geomagnetic cutoff does not allow us to resolve the time structure of the fluxes.

To match with the high statistics, we have performed extensive systematic studies as in Ref. [32]. These uncertainties affect all time bins in the same way. The relative systematic uncertainty on the flux is below $2.5 \%$ for both electrons and positrons for all energies. The main contribution to this small systematic error is from the uncertainty on the effective acceptance for electrons and positrons at energies below $2 \mathrm{GeV}$ and from charge confusion for positrons above $2 \mathrm{GeV}$. Charge confusion occurs when an electron is reconstructed as a positron and vice versa; for details see Ref. [32]. For all Bartels rotations and for all energies, the systematic error on the positron flux is smaller than the statistical error; for the electron flux this is the case above $10 \mathrm{GeV}$.

The uncertainty on the absolute energy scale of the ECAL [32] is $4.3 \%$ at $1 \mathrm{GeV}$, decreasing to $2 \%$ in the range $10-50 \mathrm{GeV}$. This is treated as an uncertainty of the bin boundaries. The bin widths $\Delta E$ are chosen to be at least 2 times the energy resolution to minimize migration effects. With the high statistics of six years, the algorithms used to determine the energy calibration of the ECAL have been optimized compared to Refs. [26,32] leading to energydependent corrections $<1.5 \%$ of the energy scale.

The time stability of the energy scale is monitored using the ratio $E / p$ of the energy measured in the ECAL $E$ to the momentum measured in the tracker $p$. The momentum scale is monitored by measurements of the proton mass using the velocity measured by the RICH and the momentum measured by the tracker. The energy scale is found to be stable at the level of $0.2 \%$ for all Bartels rotations and all energies. This produces a negligible uncertainty on the fluxes and $R_{e}$.

Most importantly, several independent analyses were performed on the same data sample. The results of those analyses are consistent with the results presented in this Letter.

Results. - The results on the time-dependent primary cosmic-ray electron flux, positron flux, and their ratio $R_{e}$ are provided in the Supplemental Material [34] as functions of energy at the top of the AMS. The time-averaged electron flux, positron flux, and the ratio $R_{e}$ are shown in Fig. 1 and listed in Table I of the Supplemental Material [34]. In Fig. 1, the points are placed horizontally at $\tilde{E}$ calculated for a flux $\propto E^{-3}$ [35]. The event selection criteria in the analyses for the fluxes $\Phi_{e^{+}}, \Phi_{e^{-}}$, and the ratio $R_{e}$ were optimized independently, see Refs. [26,32]. This accounts for the minute differences between $R_{e}$ given in the tables and that calculated from $\Phi_{e^{+}} / \Phi_{e^{-}}$. The improved precision on $R_{e}$ is apparent from Table I of the Supplemental Material [34].

To search for fine structures in the energy dependence of the fluxes, the model given in Ref. [36] was compared to the data for each Bartels rotation independently. The

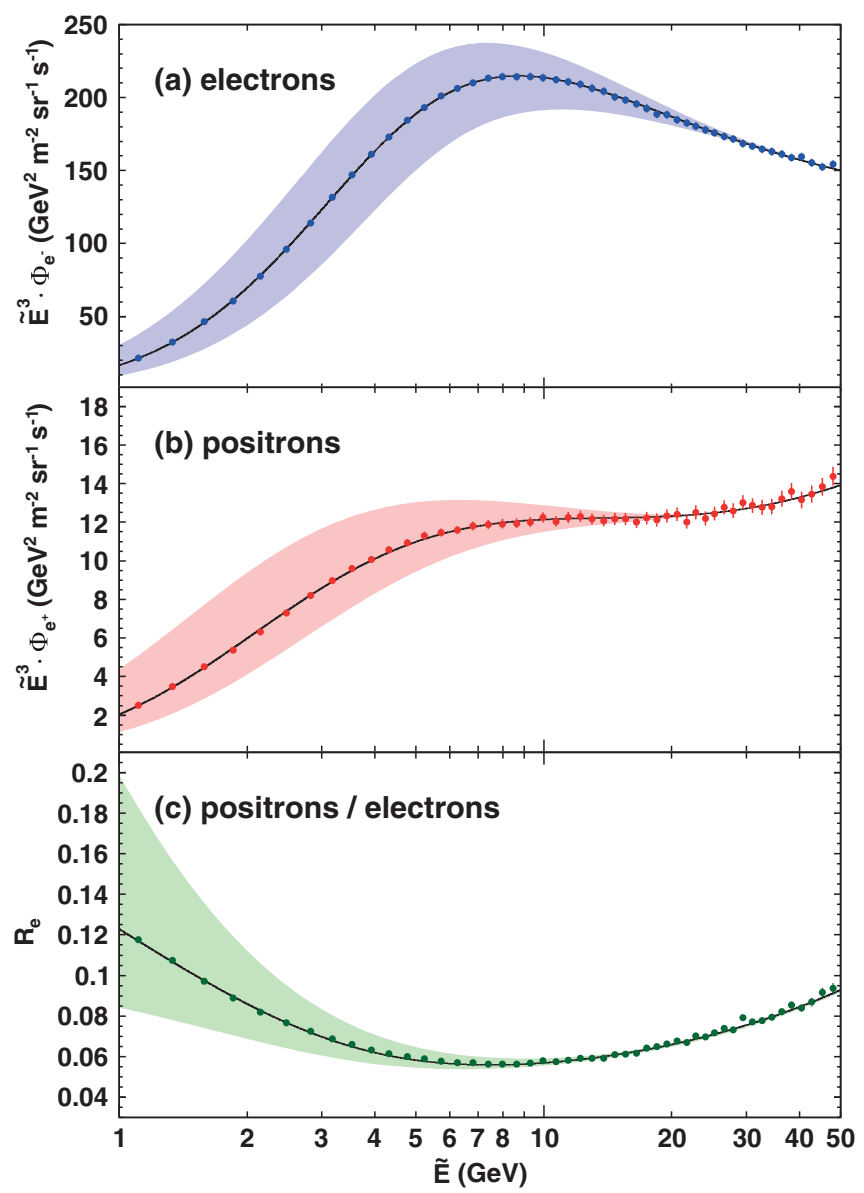

FIG. 1. The time-averaged (a) electron flux, (b) positron flux, and (c) ratio $R_{e}$. The time-variation range is indicated by the shaded regions, see text. The fit of the model in Refs. [36,37] to the time-averaged data points is shown by the black curves. The visible time variation of $R_{e}$ is evidence for charge-sign dependent solar modulation.

positron data show no additional structure, see Fig. SM 1 in the Supplemental Material [34], but the electron data reveal a model dependent residual structure in the energy range between 2 and $3 \mathrm{GeV}$, see Fig. SM 2 [34], which is stable in time and consistent with an additional smooth break $[37,38]$ in the electron spectral index $\gamma_{e^{-}}=$ $d\left(\log \Phi_{e^{-}}\right) / d(\log E)$ below $10 \mathrm{GeV}$ (Fig. SM 3 [34]), comparable to the local interstellar electron spectrum of Ref. [39]. The fits of the extended model [36] to our data yield an average $\chi^{2} /$ d.o.f. $\approx 1$ for all Bartels rotations and no fine structures in the energy spectra were found.

To visualize the magnitude of the time variations of the fluxes and of $R_{e}$, the envelopes of all fitted curves are displayed in Fig. 1 as shaded regions. The amplitude of the shaded regions decreases with increasing energy. At high energies, the statistical bin-to-bin fluctuations are larger than the time variation. As seen in Fig. 1(c), the clear time variation of $R_{e}$ is evidence for charge-sign dependent solar modulation. 


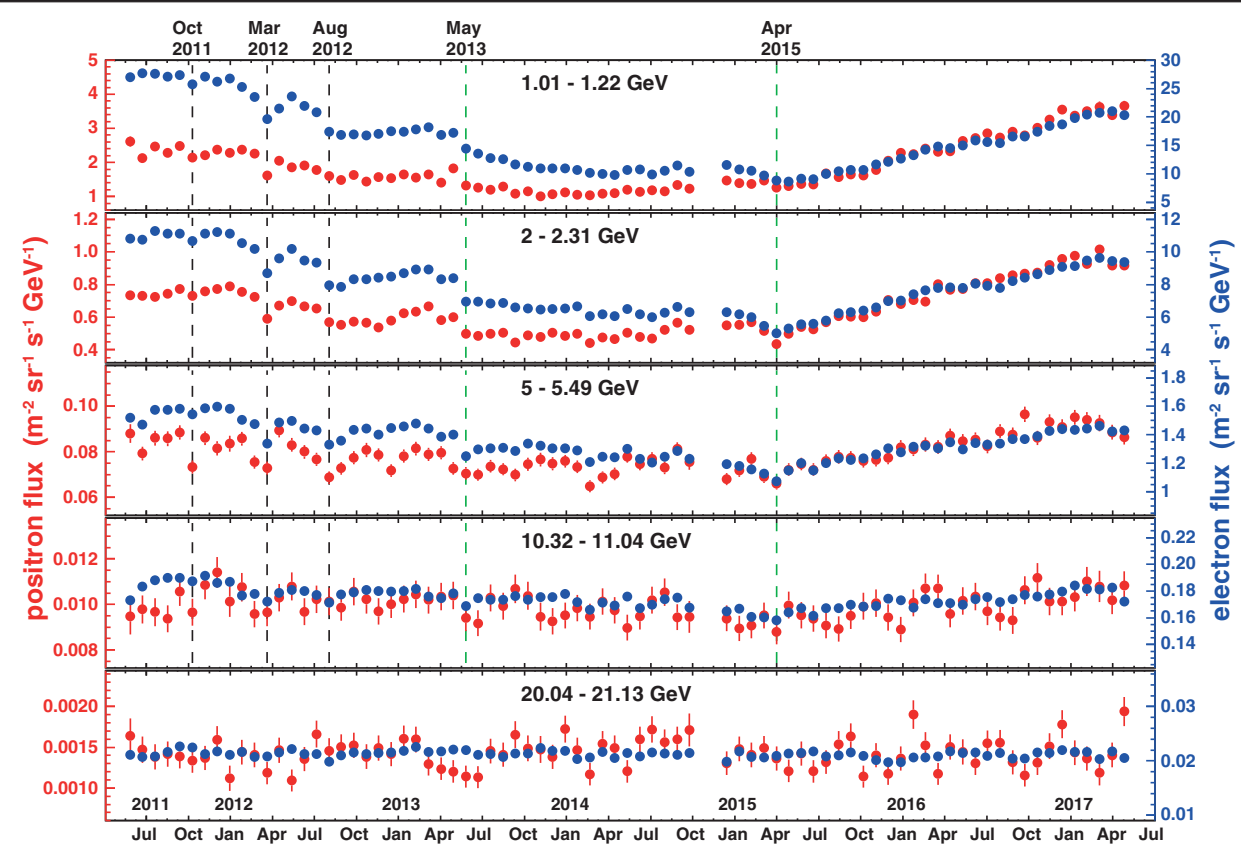

FIG. 2. Fluxes of primary cosmic-ray positrons (red, left axis) and electrons (blue, right axis) as functions of time, for five of the 49 energy bins. The error bars are the statistical uncertainties. Prominent and distinct time structures visible in both the positron spectrum and the electron spectrum and at different energies are marked by dashed vertical lines.

To study the time behavior in more detail, the fluxes are shown in Fig. 2 as a function of time for five characteristic energy bins. We find a clear evolution of the fluxes with time at low energies that gradually diminishes towards high energies. At the lowest energies, the amplitudes of both the electron flux and the positron flux change by a factor of 3 . Both fluxes exhibit profound short- and long-term variations. The short-term variations occur simultaneously in both fluxes with approximately the same relative amplitude.

On the short term of Bartels rotations, several prominent and distinct structures are observed. They are characterized by minima, visible in both the electron flux and the positron flux across the energy range below $E \lesssim 10 \mathrm{GeV}$. These are marked by dashed vertical lines in Fig. 2. Variations on short timescales have been observed at different heliographic latitudes in the combined proton and antiproton flux and also in the combined electron and positron flux [40]. A possible origin has been discussed [41].

In October 2011 and March 2012, there are sharp drops in the fluxes, followed by a quick recovery. The March 2012 event coincides with a strong Forbush decrease registered on March 8, 2012 [42].

Another drop occurred in August 2012; this was followed by an extended recovery period.

For $E \lesssim 10 \mathrm{GeV}$, May 2013 and April 2015 mark two changes in the long-term trends of the fluxes: From May 2011 to May 2013, the fluxes of both species show a trend to decrease with time. In the period around July 2013 is the time of the solar magnetic field reversal. From May 2013 to April 2015 , the flux of electrons continues to decrease, but with reduced slope, while the positron flux begins to increase.
Then, from April 2015 until May 2017, both fluxes rise steeply. The difference of the rate of the increase is related to the charge-sign dependent solar modulation $[15,43]$.

Coincident changes in both the short-term and long-term behavior have also been observed in our measurement of the proton and helium fluxes [18].

At energies above $20 \mathrm{GeV}$, neither the electron flux nor the positron flux exhibits significant time dependence.

The high statistics and continuous data presented in this Letter allow for the first time the detailed analysis of the time evolution of the spectral indices $\gamma_{e^{ \pm}}=d\left(\log \Phi_{e^{ \pm}}\right) / d(\log E)$ [32]. They are displayed at a characteristic energy of $10 \mathrm{GeV}$ in Fig. SM 4 [34]. We observe that the spectral indices for both the electrons and the positrons harden continuously with different slopes until April 2015 and then continue to soften with an identical slope. The prominent and distinct short-term structures discussed above are visible as a hardening in the spectral indices.

The long-term time structure of the data in Fig. 2 shows that the changes in relative amplitude are different for electrons and positrons. To quantify this effect, we use the ratio $R_{e}$, shown in Fig. 3. In Fig. SM 5 [34], we show our results on $R_{e}$ for all energy bins up to $5 \mathrm{GeV}$.

In $R_{e}$, the important, newly discovered short-term variations in the fluxes largely cancel, and a clear overall long-term trend appears. At low energies, $R_{e}$ is flat at first, then smoothly increases after the time of the solar magnetic field reversal, to reach a plateau at a higher amplitude.

During the extraordinarily quiet solar minimum period from 2006 to 2011, the energy and time dependence of various cosmic-ray measurements [44] including $R_{e}$ 


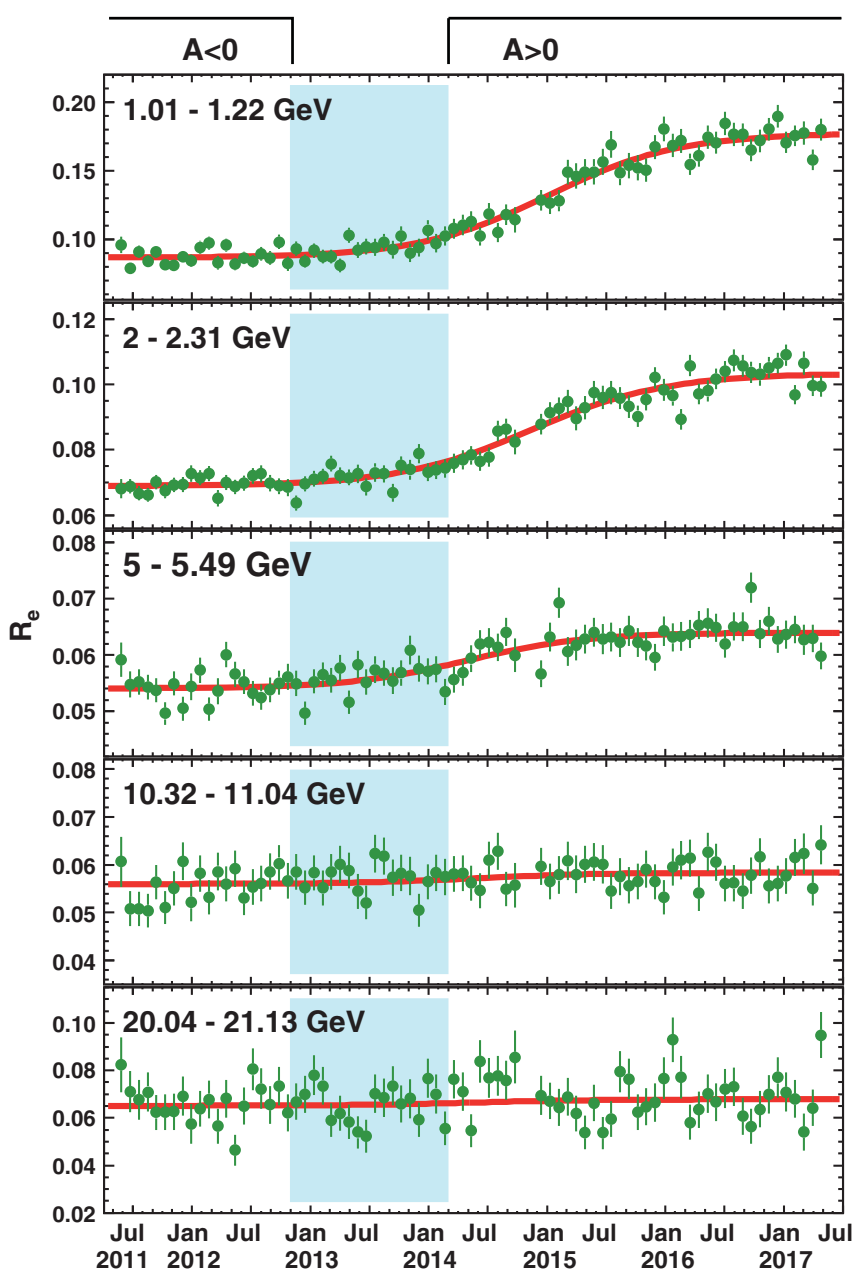

FIG. 3. The ratio $R_{e}$ of the positron flux to the electron flux as a function of time. The error bars are statistical. The best-fit parametrization according to Eq. (3) is shown by red curves. The polarity of the heliospheric magnetic field is denoted by $A<0$ and $A>0$. The period without well-defined polarity is marked by the shaded area [17].

(Fig. SM 6 [34]) are well reproduced by advanced numerical solar modulation models [22]. But for the following years covered by the new data presented in this Letter, important and large systematic discrepancies are observed in particular in $R_{e}$ (Fig. SM 6 [34]), which is sensitive to charge-sign dependent effects in the solar modulation process of galactic cosmic rays. Therefore, restricted to the time interval covered here, we use a modelindependent approach to extract the energy dependence of the quantities that characterize the observed transition in $R_{e}$. With a set of four parameters, the 3871 independent $R_{e}$ measurements as a function of energy and time can be described well with a logistic function,

$$
R_{e}(t, E)=R_{0}(E)\left[1+\frac{C(E)}{\exp \left(-\frac{t-t_{1 / 2}(E)}{\Delta t(E) / \Delta_{80}}\right)+1}\right]
$$
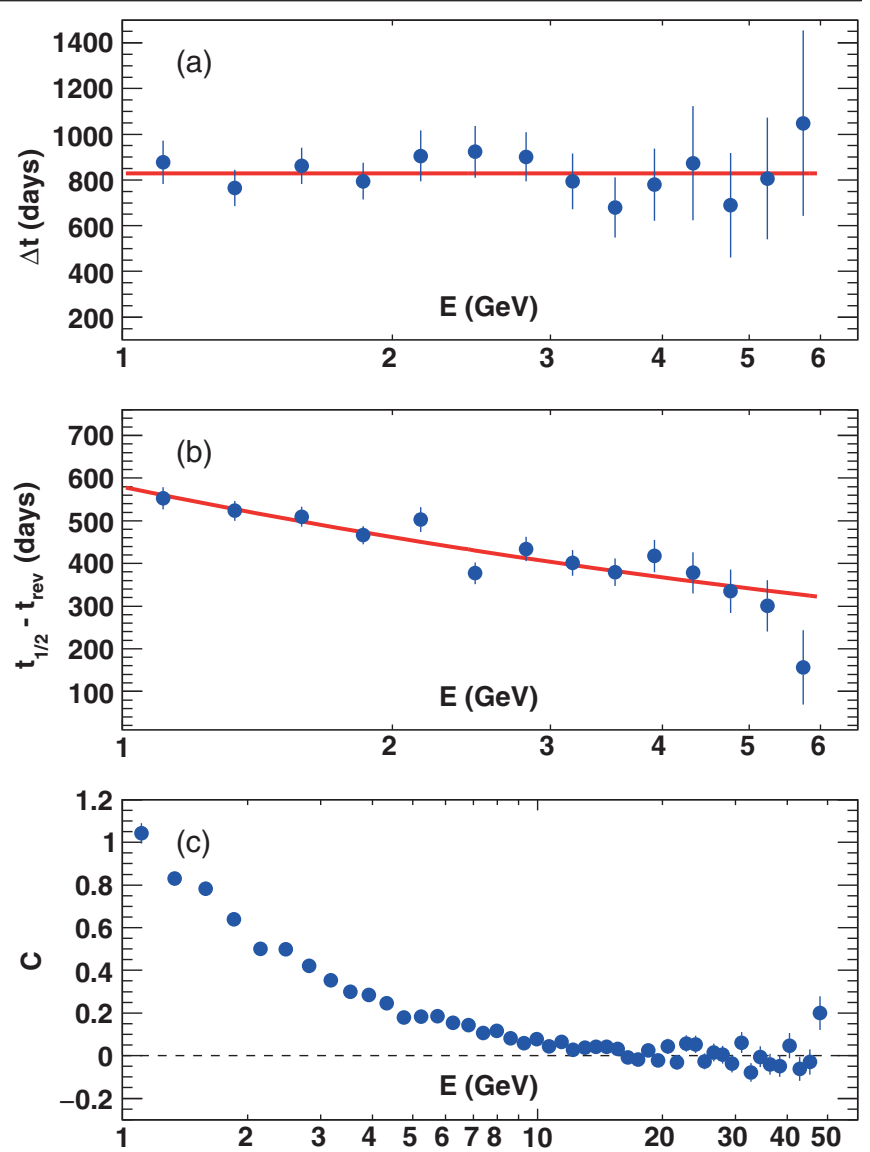

FIG. 4. Results of the fits of the parametrization in Eq. (3) to the ratio $R_{e}$ as a function of energy (blue circles): (a) $\Delta t$ and the bestfit constant value of 830 days (red line), (b) $t_{1 / 2}-t_{\text {rev }}$ with the parametrization according to Eq. (4) (red curve), (c) amplitude $C$ with a dashed line at zero to guide the eye.

At a given energy $E$, the time dependence is related to three parameters in the function: the amplitude of the transition $C$, the midpoint of the transition $t_{1 / 2}$, and the duration of the transition $\Delta t$. We choose $\Delta_{80}=4.39$, such that $\Delta t$ is the time it takes for the transition to proceed from $10 \%$ to $90 \%$ of the change in magnitude. The results of fitting Eq. (3) for each energy bin are shown in Fig. 4 . We obtain $\chi^{2} /$ d.o.f. $\approx 1$ for all fits.

The parameters $t_{1 / 2}$ and $\Delta t$ can only be determined at low energies, where the amplitude of the transition is large, see Fig. 3. As shown in Fig. 4(a), the transition duration $\Delta t$ is independent of energy, and we obtain a value of $830 \pm 30$ days.

Figure 4(b) shows the energy dependence of the delay $t_{1 / 2}$ which is well parametrized by the formula

$$
t_{1 / 2}(E)-t_{\mathrm{rev}}=\tau(E / \mathrm{GeV})^{\rho},
$$

where we choose $t_{\text {rev }}$ to be the effective time of the reversal of the solar magnetic field. For the value of $t_{\text {rev }}$, we use July 1, 2013, the center of the period without well-defined polarity [17]. The parameters used to describe the time and 
energy dependence of $R_{e}$ in Eqs. (3) and (4) are illustrated in Fig. SM 7 [34]. A fit of Eq. (4) yields the parameter $\rho=-0.33 \pm 0.04$ (stat) ${ }_{-0.15}^{+0.08}$ (syst) and the amplitude $\tau=$ $580 \pm 19$ (stat) \pm 136 (syst) days, and the value of $t_{1 / 2}$ changes by $260 \pm 30$ days from 1 to $6 \mathrm{GeV}$. The systematic uncertainties are due to the uncertainty in $t_{\text {rev }}$. This is an important and unexpected energy dependence of $t_{1 / 2}$ and reflects the different response of cosmic-ray particles and antiparticles to changing modulation conditions.

To study the amplitude $C$ in Fig. 4(c), we have fixed $\Delta t$ to its average value of 830 days and we use the value of $t_{1 / 2}$ calculated from Eq. (4) for energies above $6 \mathrm{GeV}$. At high energies, the fit result for the amplitude depends only weakly on the choice of the values for $\Delta t$ and $t_{1 / 2}$. As seen in Fig. 4(c), the amplitude $C$ is close to 1 at $E=1 \mathrm{GeV}$ and decreases smoothly with energy. This is in qualitative agreement with the expectation from solar modulation models including drift effects [41] and with the results from Refs. [13-16]. Above $20 \mathrm{GeV}$, the amplitude is consistent with zero.

In conclusion, for the first time, the charge-sign dependent modulation during solar maximum has been investigated in detail by leptons alone. We observe prominent, distinct, and coincident structures in both the positron flux and the electron flux on a time scale of months. These structures are not visible in the $e^{+} / e^{-}$flux ratio. We also observe the existence of a long-term feature in the $e^{+} / e^{-}$ flux ratio, namely, a smooth transition from one value to another, after the polarity reversal of the solar magnetic field. The duration of the transition is measured to be $830 \pm$ 30 days, independent of energy. The transition magnitude is decreasing as a function of energy, consistent with expectations from solar modulation models including drift effects. The midpoint of the transition relative to the polarity reversal of the solar magnetic field changes by $260 \pm 30$ days from 1 to $6 \mathrm{GeV}$. These high-statistics, precision data on positrons and electrons provide accurate input to the understanding of solar modulation.

We thank former NASA Administrator Daniel S. Goldin for his dedication to the legacy of the ISS as a scientific laboratory and his decision for NASA to fly AMS as a DOE payload. We also acknowledge the continuous support of the NASA leadership including Charles Bolden and William H. Gerstenmaier and of the JSC and MSFC flight control teams which has allowed AMS to operate optimally on the ISS for over six years. We are grateful for the support of Jim Siegrist and his staff of the DOE including resources from the National Energy Research Scientific Computing Center under Contract No. DE-AC02-05CH11231. We also acknowledge the continuous support from MIT and its School of Science, Michael Sipser, Marc Kastner, Ernest Moniz, Richard Milner, and Boleslaw Wyslouch. Research supported by São Paulo Research Foundation (FAPESP) Grants No. 2014/19149-7, No. 2015/50378-5, and No. 2016/ 10222-9, Brazil; CAS, NSFC, MOST, the provincial governments of Shandong, Jiangsu, Guangdong, and the China Scholarship Council, China; Action H2020 MSCAIF-2015 under Grant No. 707543-MAtISSE, European Union; the Finnish Funding Agency for Innovation (Tekes) Grants No. 40361/01 and No. 40518/03 and the Academy of Finland Grant No. 258963, Finland; CNRS/ IN2P3, CNES, Enigmass, and the ANR, France; Pascale Ehrenfreund, DLR under Grant No. 50001403 and JARAHPC under Project No. JARA0052, Germany; INFN and ASI under ASI-INFN Agreements No. 2013-002-R.0 and No. 2014-037-R.0, Italy; CHEP and NRF under Grants No. NRF-2009-0080142 and No. NRF-2012-010226 at Kyungpook National University and No. NRF-2013004883 at Ewha Womans University, Korea; the Consejo Nacional de Ciencia y Tecnología and UNAM, Mexico; FCT under Grant No. PTDC/FIS/122567/2010, Portugal; CIEMAT, IAC, CDTI, and SEIDI-MINECO under Grants No. ESP2015-71662-C2-(1-P/2-P), No. SEV-2015-0548, No. MDM-2015-0509, and No. RyC-2013-14660, Spain; the Swiss National Science Foundation (SNSF), federal and cantonal authorities, Switzerland; Academia Sinica and the Ministry of Science and Technology (MOST) under Grants No. 103-2112-M-006-018-MY3, No. 105-2112-M-001-003, and No. CDA-105-M06, former Presidents of Academia Sinica Yuan-Tseh Lee and Chi-Huey Wong and former Ministers of MOST Maw-Kuen Wu and Luo-Chuan Lee, Taiwan; the Turkish Atomic Energy Authority under Grant No. 2017TEAK(CERN)A5.H6.F2-15, Turkey; and NSF Grants No. 14255202 and No. 1551980, Wyle Laboratories Grant No. 2014/T72497, and NASA NESSF Grant No. HELIO15F-0005, USA. We gratefully acknowledge the strong support from CERN including Rolf-Dieter Heuer and Fabiola Gianotti, from the CERN IT department and Bernd Panzer-Steindel, and from the European Space Agency including Johann-Dietrich Wörner and Simonetta Di Pippo. We are grateful for important physics discussions with Fiorenza Donato, Jonathan Ellis, Jonathan Feng, Igor Moskalenko, Michael Salamon, Subir Sarkar, Joachim Trümper, Michael S. Turner, and Steven Weinberg.

\footnotetext{
${ }^{a}$ Also at ASI, I-00133 Roma, Italy.

${ }^{\mathrm{b}}$ Also at ASI Space Science Data Center (SSDC), I-00133 Roma, Italy; Present address: University of Sassari, I-07100 Sassari, Italy.

${ }^{c}$ Also at Wuhan University, Wuhan, 430072, China.

${ }^{\mathrm{d}}$ Also at Sun Yat-Sen University (SYSU), Guangzhou, 510275, China.

eAlso at ASI Space Science Data Center (SSDC), I-00133 Roma, Italy.

${ }^{\mathrm{f}}$ Also at ASI Space Science Data Center (SSDC), I-00133 Roma, Italy; Present address: INFN Sezione di Trieste, I-34149, Trieste, Italy.

${ }^{\mathrm{g}}$ Also at Nankai University, Tianjin 300071, China.

${ }^{\mathrm{h}}$ Also at Institute of Theoretial Physics, Chinese Academy of Sciences, Beijing, 100190, China.
} 
[1] H. V. Cane, Space Sci. Rev. 93, 55 (2000).

[2] M. S. Potgieter, J. A. Le Roux, L. F. Burlaga, and F. B. McDonald, Astrophys. J. 403, 760 (1993).

[3] M. S. Potgieter, Living Rev. Solar Phys. 10, 3 (2013).

[4] A. W. Strong and I. V. Moskalenko, Astrophys. J. 509, 212 (1998).

[5] C. Evoli, D. Gaggero, A. Vittino, G. Di Bernardo, M. Di Mauro, A. Ligorini, P. Ullio, and D. Grasso, J. Cosmol. Astropart. Phys. 02 (2017) 015.

[6] D. Maurin, F. Donato, R. Taillet, and P. Salati, Astrophys. J. 555, 585 (2001).

[7] A. Putze, L. Derome, and D. Maurin, Astron. Astrophys. 516, A66 (2010).

[8] M. S. Potgieter, Adv. Space Res. 53, 1415 (2014).

[9] W. R. Webber, J. C. Kish, and D. A. Schrier, Proceedings of the 18th International Cosmic Ray Conference, Vol. 3, Bangalore, India (Tata Institute, Mumbai, India, 1983).

[10] J. M. Clem, D. P. Clements, J. Esposito, P. Evenson, D. Huber, J. L'Heureux, P. Meyer, and C. Constantin, Astrophys. J. 464, 507 (1996).

[11] K. Abe et al., Phys. Lett. B 670, 103 (2008).

[12] O. Adriani et al., Phys. Rev. Lett. 116, 241105 (2016).

[13] B. Heber et al., J. Geophys. Res. 107, 1274 (2002).

[14] S. E. S. Ferreira and M. S. Potgieter, Astrophys. J. 603, 744 (2004).

[15] B. Heber, A. Kopp, J. Gieseler, R. Müller-Mellin, H. Fichtner, K. Scherer, M. S. Potgieter, and S. E. S. Ferreira, Astrophys. J. 699, 1956 (2009).

[16] B. Heber, Space Sci. Rev. 176, 265 (2013).

[17] X. Sun, J. T. Hoeksema, Y. Liu, and J. Zhao, Astrophys. J. 798, 114 (2015).

[18] M. Aguilar et al. (AMS Collaboration), preceding Letter, Phys. Rev. Lett. 121, 051101 (2018).

[19] For example, I. Cholis and D. Hooper, Phys. Rev. D 88, 023013 (2013).

[20] There are many models for the origin and propagation of cosmic rays. An overview and further references are given, e.g., in a recent review by R. Cowsik, Annu. Rev. Nucl. Part. Sci. 66, 297 (2016).

[21] M. Cirelli, D. Gaggero, G. Giesen, M. Taoso, and A. Urbano, J. Cosmol. Astropart. Phys. 12 (2014) 045.

[22] N. Tomassetti, M. Orcinha, F. Barao, and B. Bertucci, Astrophys. J. Lett. 849, L32 (2017).

[23] V. Di Felice, R. Munini, E. E. Vos, and M. S. Potgieter, Astrophys. J. 834, 89 (2017).

[24] M. S. Potgieter and E. E. Vos, Astron. Astrophys. 601, A23 (2017).

[25] R. Manuel, S. E. S. Ferreira, and M. S. Potgieter, Sol. Phys. 289, 2207 (2014).

[26] L. Accardo et al., Phys. Rev. Lett. 113, 121101 (2014).

[27] A. W. Strong, I. V. Moskalenko, and V. S. Ptuskin, Annu. Rev. Nucl. Part. Sci. 57, 285 (2007).

[28] M. Jubelgas, V. Springel, T. Enßlin, and C. Pfrommer, Astron. Astrophys. 481, 33 (2008).

[29] M. Zhang, P. Zuo, and N. Pogorelov, Astrophys. J. 790, 5 (2014).

[30] A. Kounine, Int. J. Mod. Phys. E 21, 1230005 (2012); S. Rosier-Lees, Proceedings of Astroparticle Physics TEVPA/ IDM, Amsterdam, 2014 (unpublished); S. Ting, Nucl. Phys. B,
Proc. Suppl. 243-244, 12 (2013); S.-C. Lee, Proceedings of the 20th International Conference on Supersymmetry and Unification of Fundamental Interactions (SUSY 2012), Beijing, 2012 (unpublished); M. Aguilar, Proceedings of the XL International Meeting on Fundamental Physics, Centro de Ciencias de Benasque Pedro Pascual, 2012 (unpublished); S. Schael, Proceedings of the 10th Symposium on Sources and Detection of Dark Matter and Dark Energy in the Universe, Los Angeles, 2012 (unpublished); B. Bertucci, Proc. Sci., EPS-HEP (2011) 67; M. Incagli, AIP Conf. Proc. 1223, 43 (2010); R. Battiston, Nucl. Instrum. Methods Phys. Res., Sect. A 588, 227 (2008).

[31] J. Allison et al., IEEE Trans. Nucl. Sci. 53, 270 (2006); S. Agostinelli et al., Nucl. Instrum. Methods Phys. Res., Sect. A 506, 250 (2003).

[32] M. Aguilar et al., Phys. Rev. Lett. 113, 121102 (2014).

[33] At low energies, multiple scattering distorts the particle trajectory extrapolated from the tracker into the TRD. We improve the reconstruction of the trajectory by incorporating the coordinate measurements from the TRD. This increases the effective acceptance by $40 \%$ at $1 \mathrm{GeV}$, and by $10 \%$ at $5 \mathrm{GeV}$. Above $8 \mathrm{GeV}$ the improvement is negligible. $\mathrm{N}$. Zimmermann, Ph.D. thesis, RWTH Aachen University (to be published); See also M. Graziani, Ph.D. thesis, Università degli Studi di Perugia (2015), https://cds.cern.ch/record/ 2278596.

[34] See Supplemental Material at http://link.aps.org/ supplemental/10.1103/PhysRevLett.121.051102 for additional figures and for the tabulated results on the electron flux, the positron flux, and the ratio $R_{e}$ as functions of energy at the top of AMS time-averaged from May 2011 to May 2017 and for each Bartels rotation. Data for Bartels rotations 2472 and 2473 are absent because this period was devoted to detector studies. The data can also be downloaded in different formats from the ASI cosmicray database at https://tools.asdc.asi.it/CosmicRays/.

[35] G. D. Lafferty and T. R. Wyatt, Nucl. Instrum. Methods Phys. Res., Sect. A 355, 541 (1995). We have used Eq. (6) with $\tilde{E} \equiv x_{l w}$.

[36] L. Ali Cavasonza, H. Gast, M. Krämer, M. Pellen, and S. Schael, Astrophys. J. 839, 36 (2017). The model is defined in Eq. (2a) for the positron flux and Eq. (2b) for the electron flux.

[37] C. Corti, V. Bindi, C. Consolandi, and K. Whitman, Astrophys. J. 829, 8 (2016). The prescription from Eq. (3) has been used to add a second smooth break at low energy for the electron flux in Eq. (2b) of Ref. [36].

[38] A. W. Strong, E. Orlando, and T. R. Jaffe, Astron. Astrophys. 534, A54 (2011).

[39] M. S. Potgieter, E. E. Vos, R. Munini, M. Boezio, and V. Di Felice, Astrophys. J. 810, 141 (2015).

[40] B. Heber, J. B. Blake, C. Paizis, V. Bothmer, H. Kunow, G. Wibberenz, R. A. Burger, and M. S. Potgieter, AIP Conf. Proc. 528, 357 (2000).

[41] M. S. Potgieter, Adv. Space Res. 13, 239 (1993).

[42] A. Cheminet, G. Hubert, V. Lacoste, D. Maurin, and L. Derome, J. Geophys. Res. Space Phys. 118, 7488 (2013).

[43] M. S. Potgieter, R. A. Burger, and S. E. S. Ferreira, Space Sci. Rev. 97, 295 (2001).

[44] M. S. Potgieter, Adv. Space Res. 60, 848 (2017). 